\title{
Selective Determination of Pyridine Alkaloids in Tobacco by PFTBA Ions/Analyte Molecule Reaction Ionization Ion Trap Mass Spectrometry
}

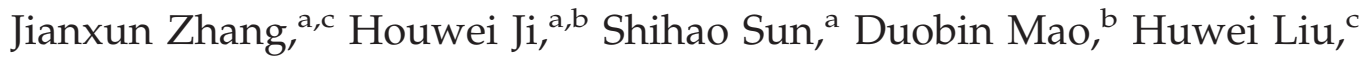 \\ and Yinlong Guo ${ }^{\text {a }}$ \\ a Shanghai Mass Spectrometry Center, Shanghai Institute of Organic Chemistry, Chinese Academy of \\ Science, Shanghai, China \\ ${ }^{\mathrm{b}}$ Food and Biological Engineering Institute, Zhengzhou University of Light Industry, Zhengzhou, China \\ ${ }^{\mathrm{c}}$ College of Chemistry and Molecular Engineering, Peking University, Beijing, China
}

The application of perfluorotributylamine (PFTBA) ions/analyte molecule reaction ionization for the selective determination of tobacco pyridine alkaloids by ion trap mass spectrometry (IT-MS) is reported. The main three PFTBA ions $\left(\mathrm{CF}_{3}{ }^{+}, \mathrm{C}_{3} \mathrm{~F}_{5}{ }^{+}\right.$, and $\left.\mathrm{C}_{5} \mathrm{~F}_{10} \mathrm{~N}^{+}\right)$are generated in the external source and then introduced into ion trap for reaction with analytes. Because the existence of the tertiary nitrogen atom in the pyridine makes it possible for PFTBA ions to react smoothly with pyridine and forms adduct ions, pyridine alkaloids in tobacco were selectively ionized and formed quasi-molecular ion $[\mathrm{M}+\mathrm{H}]^{+}$and adduct ions, including $[\mathrm{M}+69]^{+},[\mathrm{M}+$ $131]^{+}$, and $[\mathrm{M}+264]^{+}$, in IT-MS. These ions had distinct abundances and were regarded as the diagnostic ions of each tobacco pyridine alkaloid for quantitative analysis in selected-ion monitoring mode. Results show that the limit of detection is $0.2 \mu \mathrm{g} / \mathrm{mL}$, and the relative standard deviations for the seven alkaloids are in the range of $0.71 \%$ to $6.8 \%$, and good recovery of $95.6 \%$ and $97.2 \%$. The proposed method provides substantially greater selectivity and sensitivity compared with the conventional approach and offers an alternative approach for analysis of tobacco alkaloids. (J Am Soc Mass Spectrom 2007, 18, 1774-1782) (C) 2007 American Society for Mass Spectrometry

$\mathrm{T}$ Tobacco contains a number of structurally related pyridine alkaloids. These alkaloids have been widely recognized for their contributions to organoleptic properties of cigarette smoke [1]. Among those alkaloids in tobacco, nicotine, and the minor alkaloids are all pharmacologically active. However, the minor alkaloids are less potent than nicotine [2]. On the other hand, these alkaloids are of great interests due to their combination with nitrate during curing and processing, which leads to the formation of the carcinogenic, tobacco-specific nitrosamines [3]. In tobacco, the major pyridine alkaloid is nicotine, which accounts for $\sim 95 \%$ of the total alkaloid fraction. Nornicotine and anatabine are the two most abundant minor pyridine alkaloids present in roughly equal amounts, together accounting for $\sim 4 \%$ to $5 \%$ of total alkaloids [4]. Moreover, there are some minor pyridine alkaloids in tobacco, such as anabasine, myosmine, cotinine, and 2, 3'-bipyridyl, etc, which are present at smaller amounts [5]. Thus, data on the contents of total alkaloids and amounts of individual components are important and necessary in evaluating tobacco products for potential biochemical activity and marketing quality. So the

Address reprint requests to Dr. Y. L. Guo, Shanghai Mass Spectrometry Center, Shanghai Institute of Organic Chemistry, Chinese Academy of Science, Shanghai 200032, China. E-mail: ylguo@mail.sioc.ac.cn quantitative analysis of tobacco alkaloids has always been of great interest to tobacco scientists.

The concomitant presence of high levels of nicotine and lower levels of other minor alkaloids in the tobacco, along with numerous other chemical compounds, imparts a significant challenge for developing a fast and accurate quantitative method for the analysis of individual alkaloids. The current reported methods have mainly focused on the study of sample preparation and selection of a chromatogram instrument. High-performance liquid chromatography (HPLC) method measuring four alkaloids was introduced after an extensive extraction process [6]. However, HPLC method is limited for alkaloids analysis in tobacco because of the lower $\mathrm{pH}$ range for tobacco alkaloid analysis and the lower sensitivity compared with gas chromatography (GC). In recent years, capillary zone electrophoresis (CZE) has been used and developed for tobacco alkaloids analysis [7-9]. However, GC is still most commonly used for quantitation of tobacco alkaloids [10-12]. Additionally, a selective detector, nitrogenphosphorus detector, is frequently used to increase the sensitivity for trace nitrogen-containing components [13]. Before chromatographic analysis, alkaloids have to be extracted from the sample matrix. Different sample preparation techniques have been used, including traditional Soxhlet extraction [14], ultrasonication [15], 
solid-phase microextraction (SPME) [10, 16], and cloud point extraction (CPE) [17]. These methods are timeconsuming, laborious, and cumbersome. SPME suffers from matrix effects and fiber aging, and CPE, although more selective, has lower reproducibility and sensitivity. Therefore, it is necessary to develop a new method for fast and accurate analysis of tobacco alkaloids.

Chemical ionization mass spectrometry (CI-MS) is a widely used analytical technique, which is recognized for the improved selectivity and sensitivity that can be achieved in the detection of several compounds [18, 19]. It has been largely reported for quantitative analysis of some components in drug [20], food [21], and environmental pollutants [22] because of its enhanced selectivity and sensitivity.

The purpose of this work was to develop and validate an analytical method, based on the use of PFTBA ions/analyte molecule reaction ionization IT-MS for selectively detecting pyridine alkaloids in tobacco. In the proposed method, PFTBA reagent ions are generated in the external source through EI of reagent gas, and then drawn into the ion trap to react with analyte molecules eluting from GC column. Analyte ions produced by ion/molecule reaction ionization are held in the ion trap. Compared with conventional $\mathrm{CI}$, the ionization mode may avoid ion-molecule reactions with the neutral reagent and decrease background noise levels. In CI-MS, through the choice of reagent gas, selective ionization can be achieved, resulting in lower background noise levels and higher analytical sensitivity [22]. It was reported that pyridine in the gas phase forms selective adduct formation with PFTBA reagent ions by ion-molecule reaction. The existence of the tertiary nitrogen atom in the pyridine makes it possible for PFTBA ions to react smoothly with pyridine and form adduct ions [23]. Because most of the alkaloids in tobacco are pyridine alkaloids, they can be selectively determined by PFTBA ions/analyte molecule reaction ionization and self-chemical ionization [24, 25] in the ion trap spectrometer, and quasi-molecular ion $[\mathrm{M}+$ $\mathrm{H}]^{+}$and adduct ions, including $[\mathrm{M}+69]^{+},[\mathrm{M}+131]^{+}$, and $[\mathrm{M}+264]^{+}$, were formed. These ions were regarded as the diagnostic ions of each tobacco pyridine alkaloid for quantitative analysis in selected-ion monitoring (SIM) mode. Results indicated excellent selectivity and sensitivity.

\section{Experimental}

\section{Material and Reagents}

Tobacco powders (flue-cured tobacco and burley tobacco) were provided by Zhengzhou University of Light Industry. Nicotine, perfluorotributylamine (PFTBA) and quinaldine were purchased from Sigma. 2,3'Bipyridyl was purchased from Wako (Osaka, Japan). Mixed solution of standard samples for nicotine, nornicotine, myosmine, anabasine and cotinine was provided by China Tobacco Standardization Research Center
(Zhengzhou, China). Sodium hydroxide (A.R.), dichloromethane (A.R.), methanol (A.R.), and phosphoric acid (98\%) were obtained from Shanghai Chemical Solvent Company (Shanghai, China).

\section{Sample Preparation}

Tobacco powder $(1.0 \mathrm{~g})$ was weighed into a flask, $40 \mathrm{~mL}$ extracting solvent (prepared by mixing $\mathrm{CH}_{3} \mathrm{OH}$ and $\mathrm{CH}_{2} \mathrm{CL}_{2}$ in 1:3 volume ratio.), $5 \mathrm{~mL} 10 \%$ sodium hydroxide solution were added to quinaldine ( internal standard, $2 \mathrm{mg} / \mathrm{mL}$ in extracting solvent; $0.5 \mathrm{~mL}$ ), the flask was capped and placed in an ultrasonic bath containing water at ambient temperature. After sonication for $15 \mathrm{~min}$, the extract was filtered and concentrated to $10 \mathrm{~mL}$. One $\mu \mathrm{l}$ volume was used for analysis of GC/MS.

\section{GC/MS Analysis}

The GC/MS system consists of a Varian CP3800 GC gas chromatograph (Palo Alto, CA) and a Varian 4000 Ion Trap mass spectrometer (Varian,), equipped with a hybrid chemical ionization source in which the external ion source is installed, but the transfer line directs the sample into the ion trap, and reagent ions generated in the external source through EI can be selected and stored in the ion trap to react with analyte molecules. A VF-5 fused silica capillary column $(30 \mathrm{~m} \times 0.25 \mathrm{~mm} \times$ $0.25 \mu \mathrm{m})$ (Varian) was used. The carrier gas was helium at a flow of $1 \mathrm{~mL} \mathrm{~min}^{-1}$ in a constant-flow mode and splitless mode injection was employed. The injector temperature, trap temperature, and ion source temperature were 250,180 , and $200{ }^{\circ} \mathrm{C}$, respectively. The initial oven temperature of $80{ }^{\circ} \mathrm{C}$ was held for $1 \mathrm{~min}$ and then programmed at $10{ }^{\circ} \mathrm{C} \mathrm{min}^{-1}$ to $200{ }^{\circ} \mathrm{C}$ which was held for $2 \mathrm{~min}$, and then at $40^{\circ} \mathrm{C} \mathrm{min}^{-1}$ to $280{ }^{\circ} \mathrm{C}$, which was then held for $1 \mathrm{~min}$. The transfer line temperature was at $250{ }^{\circ} \mathrm{C}$. The ion trap mass spectrometer was operated in positive chemical ionization mode with PFTBA as reagent, and simultaneously in normal scan mode (scan range $m / z 50$ to 500 ). The ion gauge pressure was 50 $\mu$ Torr.

Compounds were identified by the mass spectra of each tobacco alkaloid in PCI mode, which was its quasi-molecular ion peak and adduct ion peak. Identification of nicotine, nornicotine, myosmine, anabasine, 2,3'-bipyridyl, and cotinine was further confirmed by use of authentic reference substances.

Quantitative analysis was performed in selected-ion monitoring (SIM) mode, and the quantitation was made with calibration curves by internal standard procedure with quinadine as internal standard. Since nicotine and 2,3'-bipyridyl were available and other alkaloids were not available, it was assumed that they have a response factor similar to 2,3'-bipyridyl (i.e., $\mathrm{F}=1.00$ was used for all compound). Two calibration solutions of standard alkaloids dissolved in dichloromethane were prepared from 0.2 to $4.0 \mathrm{mg} / \mathrm{mL}$ of nicotine and from 2.0 to 


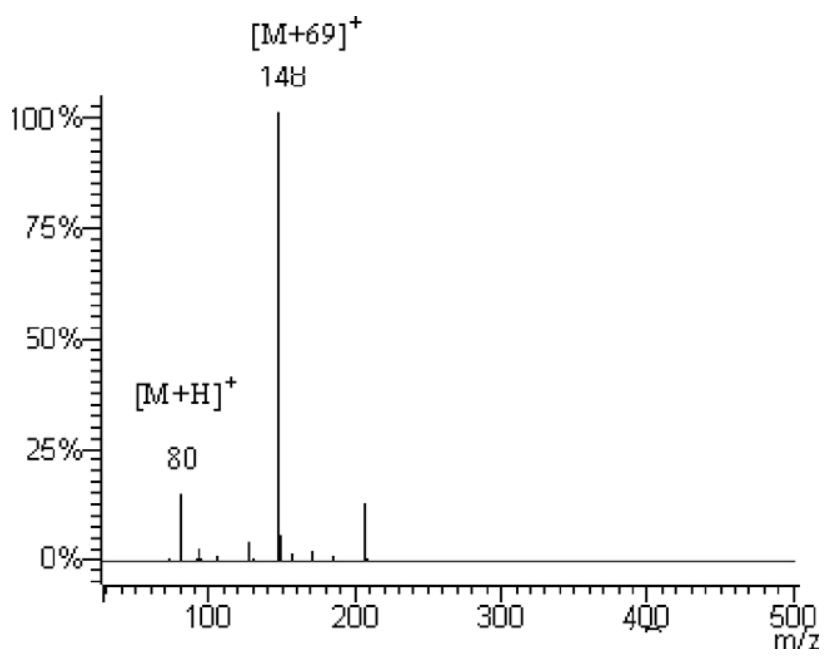

Figure 1. Mass spectra for pyridine reacting with individual reagent ion, $\mathrm{CF}_{3}{ }^{+}(69)$, in IT-MS.

$40.0 \mu \mathrm{g} / \mathrm{mL}$ of $2,3^{\prime}$-bipyridyl. The concentration of the I.S. quinaldine was $100 \mu \mathrm{g} / \mathrm{mL}$.

\section{Results and Discussion}

\section{Selection of CI Reagents}

The use of chemical ionization is undoubtedly the most common application of ion-molecule reactions in analytical mass spectrometry. Proton-transfer reagents are the most frequently used chemical ionization reagents because almost all organic analyte molecules undergo protonation, thus forming quasi-molecular ions for molecular weight determinations. The development of alternative reagents for chemical ionization has always been an area of interest for several reasons [26]: new reagents have been used for selective detection and quantification of compound in complex matrix. Methane reagent gas is commonly used in PCI mode. Due to its relatively lower proton affinity, $\mathrm{CH}_{5}{ }^{+}$of the CI reagent ions generated by methane reacts rapidly with almost every compound; the use of methane as reagent gas for analysis of the complex tobacco matrix lacks selectivity; ammonia as reagent gas forms $\mathrm{NH}_{4}{ }^{+}$adduct with sample molecule in PCI mode, especially the heterocyclics (containing $\mathrm{N}, \mathrm{O}$, et al.) and the unsaturated compounds [27]. However, ammonia is not facile. Methanol and acetone reagents are usually available, but both for analysis of tobacco alkaloids in complex tobacco matrix lack selectivity and sensitivity. Therefore, it is essential that a CI reagent for selective analysis of tobacco alkaloids in complex matrix is developed. It was reported that pyridine in the gas phase formed selective adduct formation with PFTBA reagent ions by ion-molecular reaction [23]. In this work, PFTBA is used as reagent because the three main PFTBA ions $\left(\mathrm{CF}_{3}{ }^{+}\right.$, $\mathrm{C}_{3} \mathrm{~F}_{5}{ }^{+}$, and $\mathrm{C}_{5} \mathrm{~F}_{10} \mathrm{~N}^{+}$) can selectively react with pyridine and make it ionized and form quasi-molecular ion [M + $\mathrm{H}]^{+}$and adduct ions, including $[\mathrm{M}+69]^{+},[\mathrm{M}+131]^{+}$, and $[\mathrm{M}+264]^{+}$. These ions can be regarded as the diagnostic ions of each tobacco pyridine alkaloid.

\section{Selection of the Reagent Ions and Optimizing the Reaction Time}

PFTBA as reagent mainly generates three reagent ions in the external source through electron ionization: $\mathrm{CF}_{3}{ }^{+}(69), \mathrm{C}_{3} \mathrm{~F}_{5}{ }^{+}(131)$, and $\mathrm{C}_{5} \mathrm{~F}_{10} \mathrm{~N}^{+}$(264). At first, individual reagent ion, $\mathrm{CF}_{3}{ }^{+}(69)$, was isolated and entered the ion trap to react with sample molecule (pyridine) [28], forming quasi-molecular ions $[\mathrm{M}+1]^{+}$and adduct ion $[\mathrm{M}+69]^{+}$by ion-molecule reactions (Figure 1 ). When the isolation of individual reagent ions was used for analysis of the sample, results indicated that the method had lower sensitivity. However, when all reagent ions entered the ion trap to react with the sample molecule (pyridine), the sensitivity increased two to three orders of magnitude for product ions, including quasi-molecular ions $[\mathrm{M}+\mathrm{H}]^{+}$, adduct ion $[\mathrm{M}+69]^{+}$, $[\mathrm{M}+131]^{+},[\mathrm{M}+264]^{+}$(Figure 2). The possible reason is that choice of all reagent ions enhances reagent ions intensity, resulting in increasing ionization of sample molecule. Therefore selection of all reagent ions was used for analysis of sample.

To explore the influence of reaction time on ionization of sample molecule, a series of experiments was performed to optimize the reaction time $(50,100,200$, $400,800 \mathrm{~ms}$ ). The optimal result was achieved when the reaction time was $200 \mathrm{~ms}$. The potential reason is that the ionization of sample increases as the reaction time increases. When the reaction time gets to a certain point, sample molecules are ionized completely. On the other hand, because ions have certain residence time in the source [29], exceedingly long reaction time can lead to ion waste and decrease ion intensity. An optimal reaction time of 200 ms was selected for the analysis of sample.

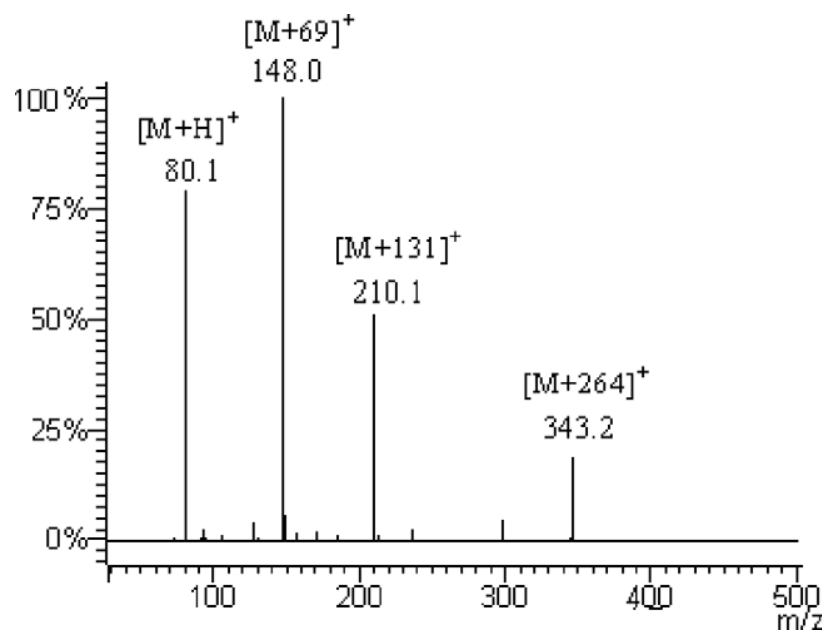

Figure 2. Mass spectra for pyridine reacting with all reagent ions in IT-MS. 


\section{Sample Preparation}

Pyridine alkaloids are present in tobacco as free bases and salts. To achieve satisfactory recovery and extraction efficiency, it is necessary to choose the optimum extraction solvent and basic or acid solution. The use of a mixture of 1:3 (vol/vol) methanol and dichloromethane resulted in satisfactory recovery was reported [13]. Thus, the mixture of 1:3 (vol/vol) methanol and dichloromethane was chosen in this work. The choice of basic or acid solution is to affect the extraction efficiency of the solvent. Basic solution helps to convert the alkaloids from the ions to free bases. Acid solution can convert the alkaloids from free bases to ions, and then the alkaloids are converted to free bases by adjusting the $\mathrm{pH}$ to $11-13$. In this work, $10 \%$ sodium hydroxide solution and $10 \%$ phosphoric acid solution were investigated for examining basic or acid effect of extraction efficiency of tobacco alkaloids. Results (Figure 3) indicated $10 \%$ sodium hydroxide solution was superior to $10 \%$ phosphoric acid solution in improving extraction
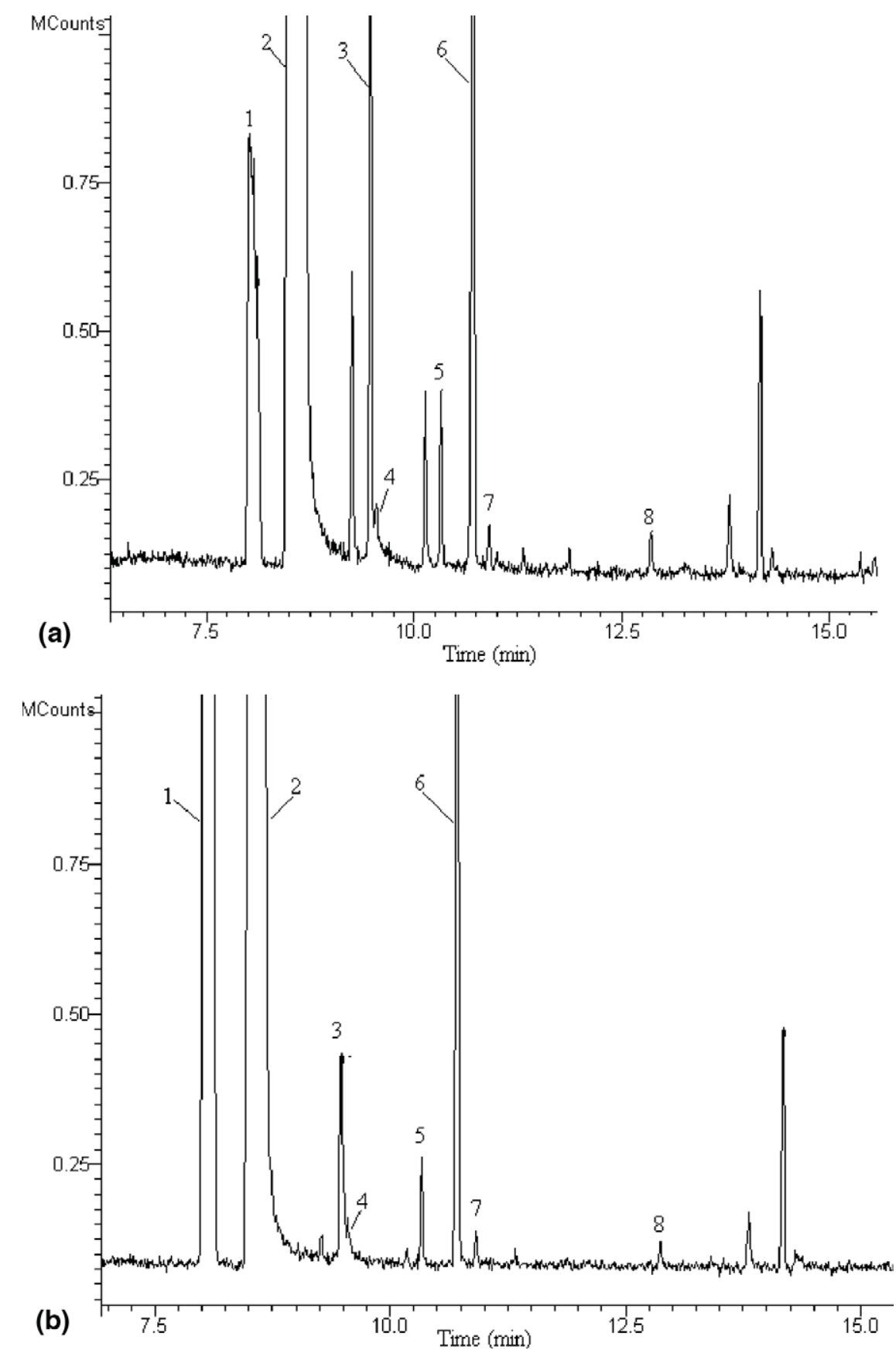

Figure 3. Total ion chromatogram (TIC) of the tobacco alkaloids obtained by the use of (a) $10 \%$ sodium hydroxide and (b) $10 \%$ phosphoric acid. (1, quinaldine; 2, nicotine; 3, nornicotine; 4 , myosmine; 5 , anabasine; 6 , anatabine; 7, 2,3'-bipyridyl; 8, cotinine). 
efficiency of tobacco alkaloids. Moreover, the procedure of the use of $10 \%$ sodium hydroxide solution was simpler and more convenient. Therefore, $10 \%$ sodium hydroxide solution was chosen for extraction of tobacco alkaloids.

\section{Qualitative Analysis Alkaloids in Tobacco} by the Proposed Method

It is well-known that chemical ionization in mass spectrometry provides mild ionization conditions that often results in formation of abundant quasi-molecular ions and adduct ions. Molecular masses of mixture components being rarely overlapped, CI enables carrying out the analyses of target compounds in the most selective way, especially the choice of reagent gas. In this wok, PFTBA as reagent was investigated for the analysis of pyridine alkaloids in tobacco. The proposed method has been described above. Experimental results indicated the method may determinate, simultaneously,
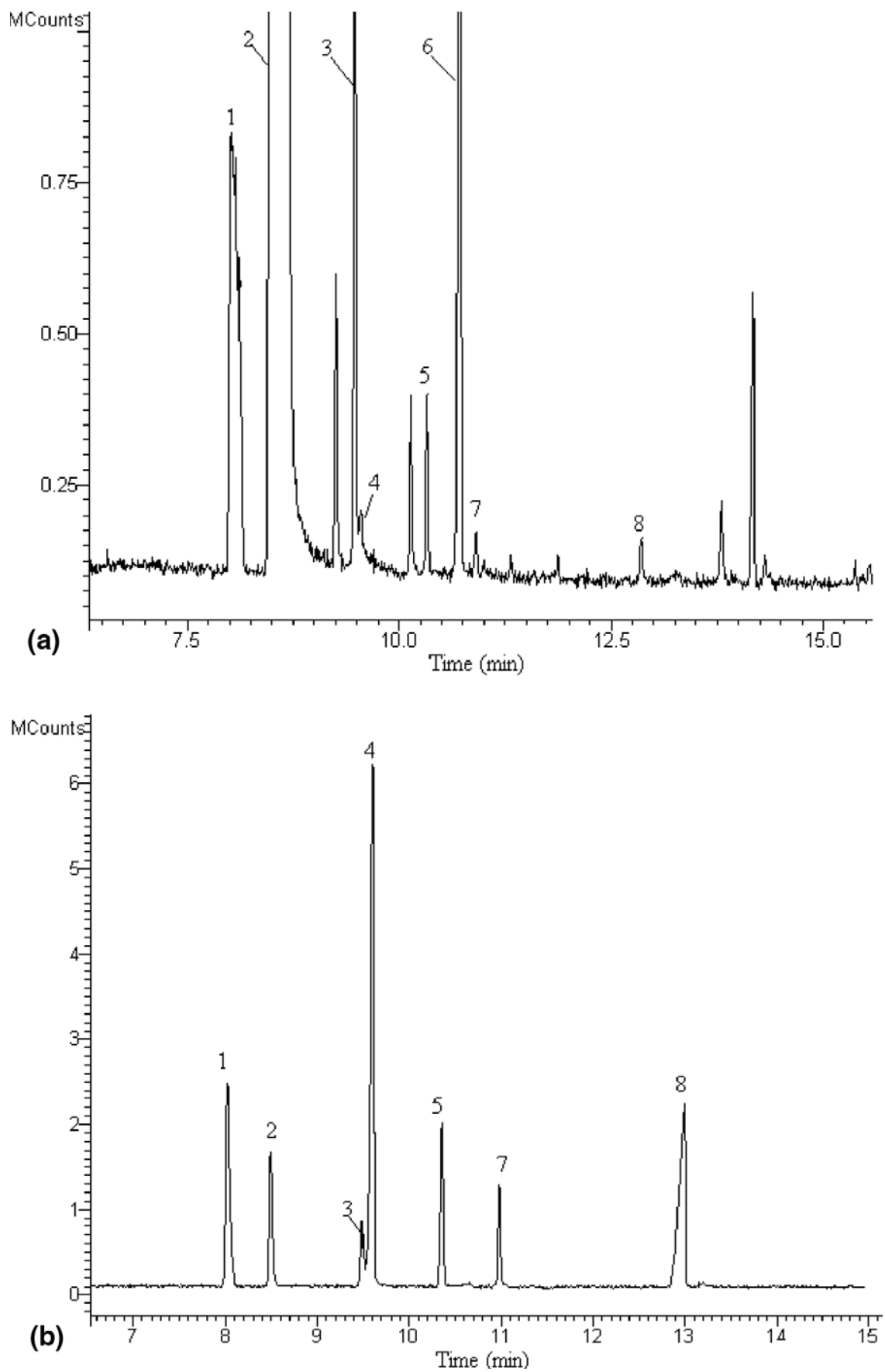

Figure 4. Total ion chromatogram (TIC) for seven tobacco alkaloids obtained by the proposed method, PFTBA as reagent: (a) burley tobacco sample; (b) mixture of internal standard and six standard tobacco alkaloids (1, quinaldine; 2 , nicotine; 3, nornicotine; 4, myosmine; 5 , anabasine; 6 , anatabine; 7, 2,3'-bipyridyl; 8, cotinine). 

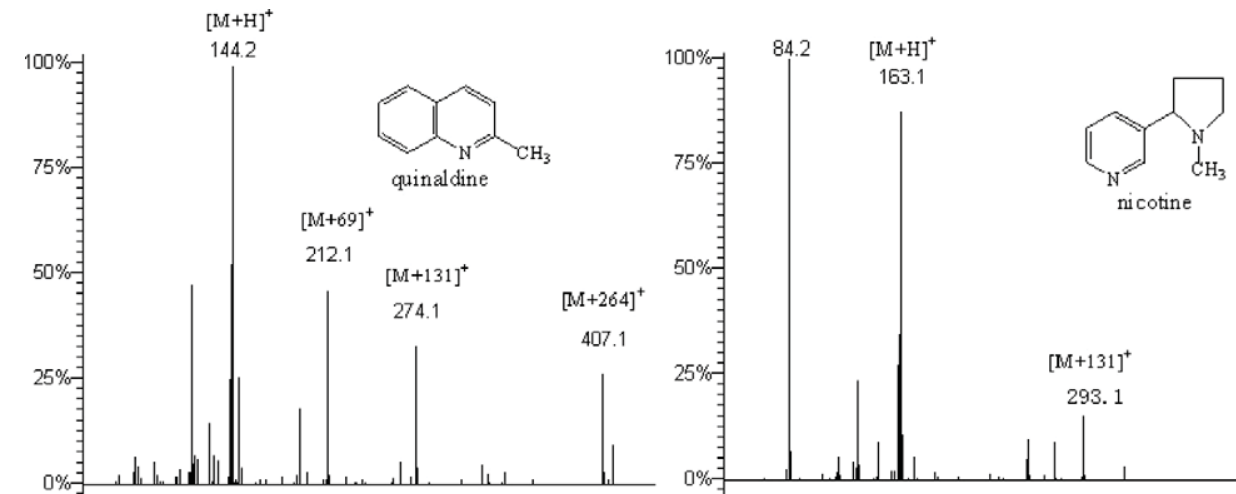

(a)

100

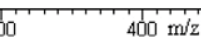

(b)
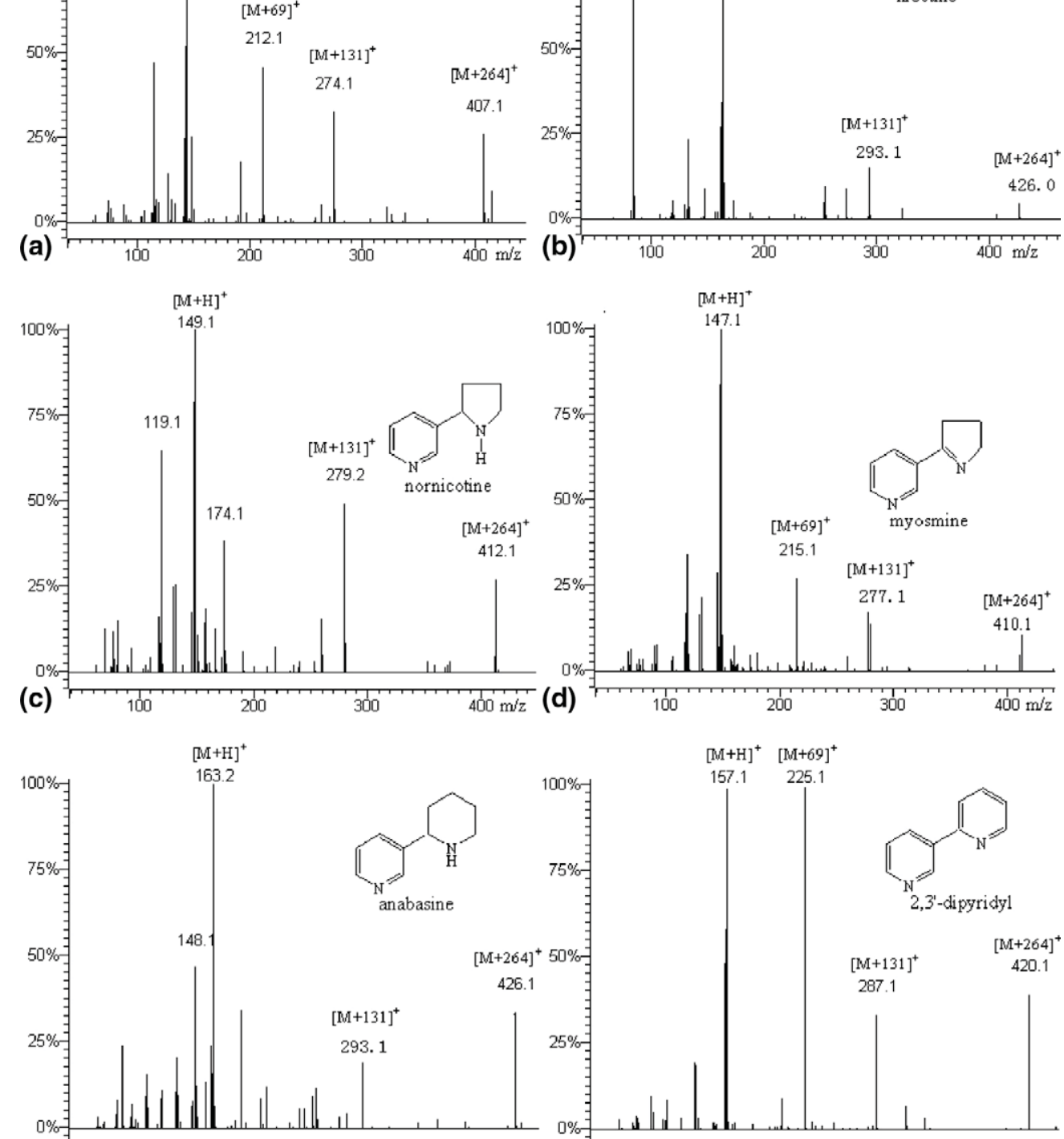

$[\mathrm{M}+\mathrm{H}]^{+}[\mathrm{M}+69]^{+}$

(d)

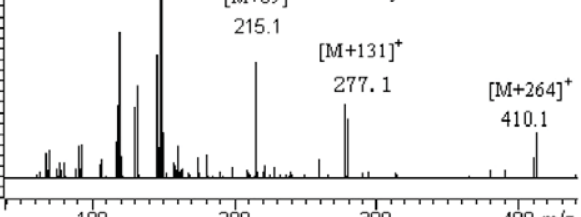

(e)
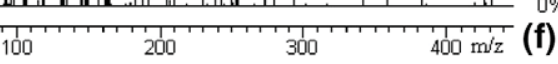

(f)
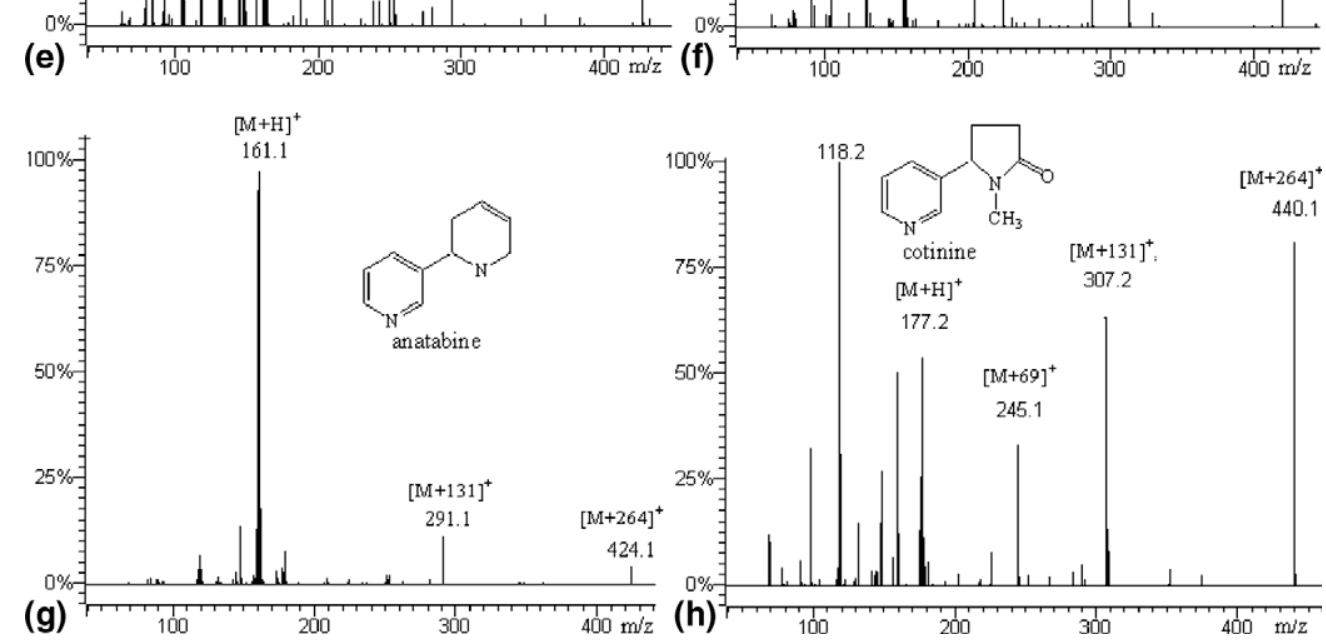

Figure 5. Mass spectra for internal standard (IS) and seven tobacco alkaloids obtained by the proposed method: (a) quinaldine; (b) nicotine; (c) nornicotine; (d) myosmine; (e) anabasine; (f) 2,3'-bipyridyl; (g) anatabine; (h) cotinine. 
seven tobacco pyridine alkaloids in complex matrix, including nicotine, nornicotine, myosmine, anabasine, anatabine, cotinine, and 2,3'-bipyridyl. The method was more sensitive and selective for the analysis of pyridine alkaloids in tobacco. Seven tobacco alkaloids were confirmed through mass spectra of individual tobacco pyridine alkaloids and standard sample retention times (Figure 4). Mass spectra of individual tobacco pyridine alkaloids showed obvious ion peak of quasi-molecular ion and adduct ions (Figure 5).

\section{Quantitative Analysis of Alkaloids in Different Tobacco Samples}

To investigate the applicability of the method, two tobacco samples (flue-cured and burley) were selected and analyzed with the proposed method. As an example, a chromatogram of tobacco sample is given in Figure 4a. The method was found to be effective for simultaneous and selective determination of nicotine and six minor alkaloids in tobacco. Furthermore, quantitative analysis of tobacco alkaloids was conducted by using the internal standard method in SIM mode and quasi-molecular ions $[\mathrm{M}+\mathrm{H}]^{+}$, adduct ion $[\mathrm{M}+69]^{+}$, $[\mathrm{M}+131]^{+}$, and $[\mathrm{M}+264]^{+}$ions were selected for monitoring ions.

Two calibration curves for nicotine and 2,3'-bipyridyl were constructed with quinaldine as internal standard in SIM mode. The method showed a satisfactory linearity for all compounds in the range of concentration study, with correlation coefficients $\left(R^{2}\right)$ of $0.9995,0.9990$ for nicotine and 2,3'-bipyridyl, respectively. The results of regression analysis for different alkaloids are presented in Table 1. The limit of detection was $0.2 \mu \mathrm{g} / \mathrm{mL}$ for 2,3'-bipyridyl.

The repeatability of the whole procedure was determined by extracting the tobacco alkaloids five times over a short time under the same conditions. The global method was evaluated for tobacco with low and high levels of alkaloids through relative standard deviations (RSD) of the results (Table 2). The results showed that the contents of tobacco alkaloids in burley tobacco were distinctly higher than in flue-cured tobacco. The recovery of the method is summarized in Table 3. The average recovery for nicotine was $97.2 \%$, with RSD of $1.7 \%$; for $2,3^{\prime}$-bipyridyl it was $95.6 \%$, with RSD of $0.8 \%$ $(n=5)$.

Table 1. Equations from regression analysis

\begin{tabular}{llcc}
\hline \multicolumn{1}{c}{ Alkaloid } & $\begin{array}{c}\text { Regression } \\
\text { equation }\end{array}$ & $\begin{array}{c}\text { Correlation } \\
\text { coefficient } \\
\left(\mathrm{R}^{2}\right)\end{array}$ & $\begin{array}{c}\text { Limit of } \\
\text { detection } \\
(\mathrm{LOD})\end{array}$ \\
\hline \hline Nicotine & $\mathrm{y}=11.29 \mathrm{x}-$ & 0.9995 & $0.3 \mu \mathrm{g} / \mathrm{mL}$ \\
2,3' 'bipyridyl & $\begin{array}{l}1.6678 \\
=0.00460 \mathrm{x}+\end{array}$ & 0.9990 & $0.2 \mu \mathrm{g} / \mathrm{mL}$ \\
& 0.00511 & & \\
\hline
\end{tabular}

$y$ : the alkaloids to internal standard peak-area ratio. $x$ : the concentration of alkaloid.
Table 2. Pyridine alkaloid contents of tobacco and relative standard deviations (RSD) of chromatographic

\begin{tabular}{lccccc}
\hline & \multicolumn{2}{c}{ Burley } & & \multicolumn{2}{c}{ Flue-cured } \\
\cline { 2 - 3 } \cline { 5 - 6 } $\begin{array}{l}\text { Tobacco pyridine } \\
\text { alkaloids }\end{array}$ & $\begin{array}{c}\text { Average } \\
(\mathrm{mg} / \mathrm{g})\end{array}$ & $\begin{array}{c}\text { RSD\% } \\
(n=5)\end{array}$ & $\begin{array}{c}\text { Average } \\
(\mathrm{mg} / \mathrm{g})\end{array}$ & $\begin{array}{c}\text { RSD\% } \\
(n=5)\end{array}$ \\
\hline \hline Nicotine & 45.29 & 3.8 & & 20.22 & 3.4 \\
Nornicotine & 0.745 & 5.5 & & 0.102 & 0.7 \\
Anabasine & 0.229 & 1.4 & & 0.0933 & 1.3 \\
Anatabine & 1.196 & 5.1 & & 0.798 & 6.2 \\
2,3'-bipyridyl & 0.0546 & 5.5 & & 0.0188 & 3.0 \\
Cotinine & 0.0497 & 1.2 & & 0.0124 & 6.8 \\
\hline
\end{tabular}

Comparison of Analysis of Pyridine Alkaloids

in Tobacco by the Proposed Method

and Conventional GC-EI-MS

Analysis of pyridine alkaloids in tobacco by the conventional GC-EI-MS was performed under the same sample preparation and condition of GC analysis with the proposed method, and total ion chromatogram (TIC) of burley tobacco and selected ion chromatogram (SIC) of 2,3'-bipyridyl and cotinine were obtained, as shown in Figure 6. The signal/noise ratios $(\mathrm{S} / \mathrm{N})$ of $2,3^{\prime}$-bipyridyl and cotinine indicated that the $\mathrm{S} / \mathrm{N}$ of the proposed method was more than the one of the GCEI-MS method. Thus, compared with the proposed method, it was found that the GC-EI-MS method showed lower selectivity and sensitivity, resulting in poorer chromatogram peak of individual tobacco alkaloid, especially minor 2,3'-bipyridyl and cotinine. The limit of detection $(\mathrm{S} / \mathrm{N}>3)$ was determined with regard to 2,3'-bipyridyl standard, analyzed with the two ionization methods.. The LOD for EI is $0.6 \mu \mathrm{g} / \mathrm{mL}$ and the one for the proposed method is $0.2 \mu \mathrm{g} / \mathrm{mL}$, which is less than the one for GC-EI-MS, depending upon the emission current and the maximum reaction time used. Therefore, it was difficult to use the GCEI-MS method for quantitative analysis of minor tobacco alkaloids. Nevertheless, because of the greater selectivity and sensitivity of the proposed method, accurate quantitative analysis of individual pyridine alkaloid in tobacco was achieved in SIM mode.

\section{Conclusions}

The present work reports that an analytical method for selective analysis of tobacco pyridine alkaloids by

Table 3. The recovery of alkaloids (nicotine and 2,3'-bipyridyl)

\begin{tabular}{|c|c|c|c|c|c|}
\hline \multirow[b]{2}{*}{$\begin{array}{l}\text { Tobacco } \\
\text { alkaloids }\end{array}$} & \multicolumn{3}{|c|}{ Concentration } & \multicolumn{2}{|c|}{$\begin{array}{l}\text { Average } \\
\text { recovery } \\
(n=5)\end{array}$} \\
\hline & $\begin{array}{l}\text { Initial } \\
(\mathrm{mg} / \mathrm{g})\end{array}$ & $\begin{array}{l}\text { Added } \\
(\mathrm{mg} / \mathrm{g})\end{array}$ & $\begin{array}{l}\text { Found } \\
(\mathrm{mg} / \mathrm{g})\end{array}$ & $\%$ & RSD \\
\hline Nicotine & 45.29 & 25.10 & 68.42 & 97.2 & $1.7 \%$ \\
\hline 2,3'-bipyridyl & 0.0546 & 0.0431 & 0.0933 & 95.6 & $2.1 \%$ \\
\hline
\end{tabular}



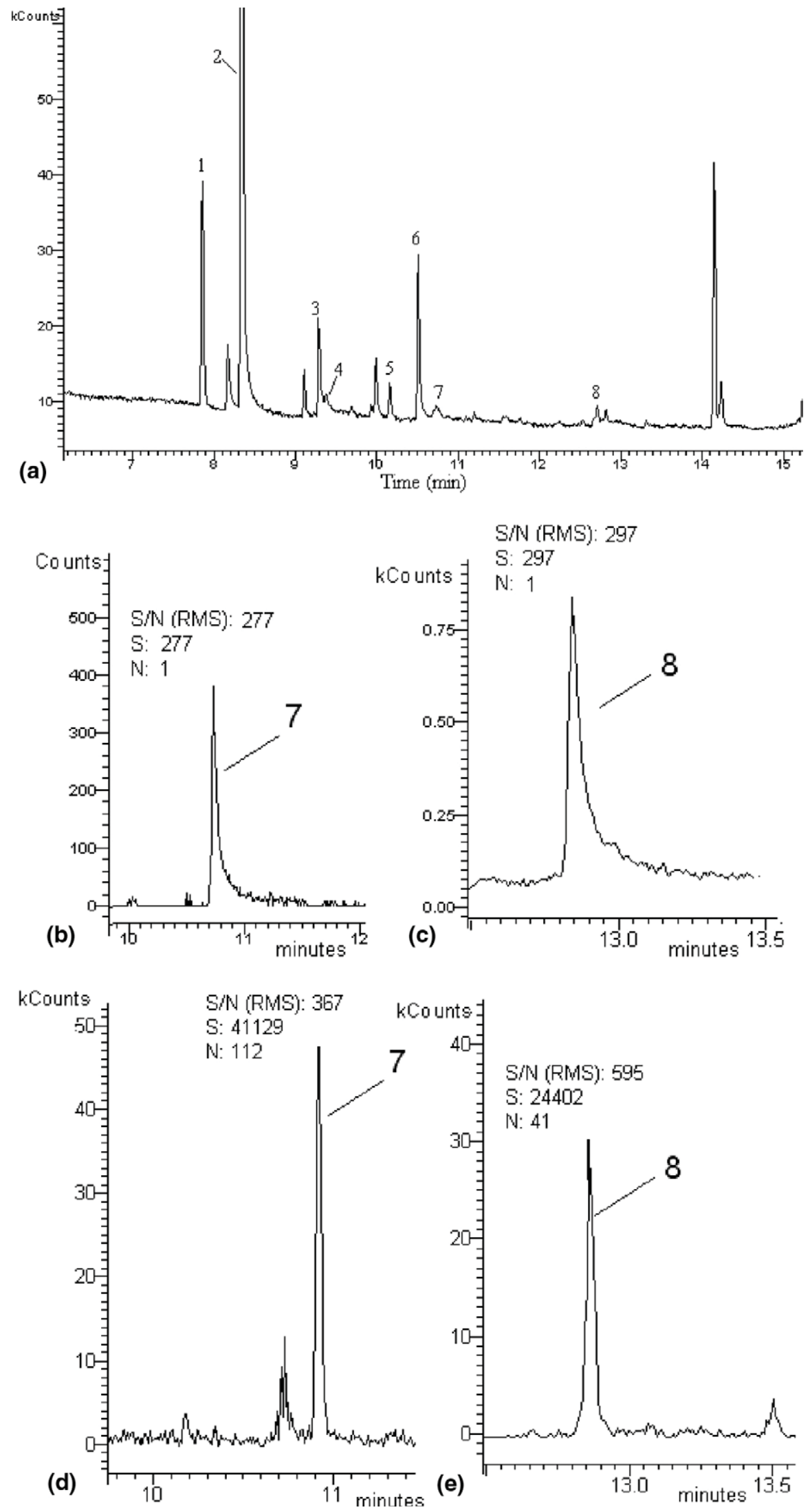

Figure 6. (a) Total ion chromatogram for seven tobacco alkaloids obtained by GC-EI-MS (1, quinaldine; 2, nicotine; 3, nornicotine; 4, myosmine; 5 , anabasine; 6 , anatabine; 7, 2,3'-bipyridyl; 8, cotinine); (b) and (c) chromatograms of 2,3'-bipyridyl and cotinine obtained under EI-SIM; (d) and (e) chromatograms of 2,3'-bipyridyl and cotinine obtained under CI-SIM. 
PFTBA ions/analyte molecule reaction ionization in the ion trap mass spectrometer was developed and successfully applied to the determination of nicotine and six minor pyridine alkaloid contents in tobacco samples. The sample preparation was selective and simple. The selective ionization of pyridine by PFTBA ions/analyte molecule reaction resulted in forming $[\mathrm{M}+69]^{+},[\mathrm{M}+$ $131]^{+},[\mathrm{M}+264]^{+}$, and $[\mathrm{M}+\mathrm{H}]^{+}$ions because of the existence of the tertiary nitrogen atom in the pyridine and is used for the analysis of compounds containing pyridines or pyridyl functional groups. It was shown that the proposed method enabled registration of mass spectra, including the distinct quasi-molecular ions and adduct ions for the pyridine alkaloids in tobacco; thus, quantitative analysis of tobacco pyridine alkaloids were performed in SIM mode and obtained lower detection limit of $0.2 \mu \mathrm{g} / \mathrm{mL}$ depending on a compound that is comparable with electron ionization (EI), yet providing ultimate sensitivity and satisfactory repeatability (RSD < $7 \%, n=5)$, and good recovery. Therefore, the proposed method is proven to be suitable for simultaneous determination of seven pyridine alkaloids in tobacco samples. Also, the method should have wide applicability to the analysis of alkaloids containing pyridyl functional group in other matrices.

\section{Acknowledgments}

The authors gratefully acknowledge the National Natural Science Foundation of China (20475059) and the Chinese Academy of Sciences (KGCX2-SW-213-04) for financial support.

\section{References}

1. Andersen, R. A.; Fleming, P. D.; Burton, H. R.; Hamiltonkmp, T. R.; Sutton, T. G. Nitrosated, Acylated, and Oxidized Pyridine Alkaloids During Storage of Smokeless Tobaccos: Effects of Moisture, Temperature, and Their Interactions. J. Agric. Food Chem. 1991, 39, 1281-1287.

2. Liu, X.; Castagnoli, K.; Van der Schyf, C. J.; Castagnoli, N., Jr. Studies on the in Vivo Biotransformation of the Tobacco Alkaloid $\beta$-Nicotyrine. Chem. Res. Toxicol. 2000, 13, 336-341.

3. Burton, H. R.; Dye, N. K.; Bush, L. P. Relationship Between TobaccoSpecific Nitrosamines and Nitrite from Different Air-Cured Tobacco Varieties. J. Agric. Food Chem. 1994, 42, 2007-2011.

4. Saitoh, F.; Noma, M.; Kawashima, N. The Alkaloid Contents of Sixty Nicotiana Species. Phytochemistry 1985, 24, 477-480.

5. Schmeltz, I.; Hoffman, D. Nitrogen-Containing Compounds in Tobacco and Tobacco Smoke. Chem. Rev. 1977, 77, 295-311.

6. Plade, J. J.; Hoffmann, D. Chemical Studies on Tobacco Smoke. LXVII. Quantitative Determination of Alkaloids in Tobacco by Liquid Chromatography. J. Liquid Chromatogr. 1980, 3, 1505-1515.

7. Yang, S. S. Evaluation of Capillary Electrophoresis for the Analysis of Nicotine and Selected Minor Alkaloids from Tobacco. Chromatographia 1995, 40, 375-378.
8. Yang, S. S.; Smetena, I.; Goldsmith, A. I. Evaluation of Micellar Electrokinetic Capillary Chromatography for the Analysis of Selected Tobacco Alkaloids. J. Chromatogr. A 1996, 746, 131-136.

9. Lochmann, H.; Bazzanella, A.; Kropsch, S.; Bachmann, K. Determination of Tobacco Alkaloids in Single Plant Cells by Capillary Electrophoresis. J. Chromatogr. A 2001, 917, 311-317.

10. Yang, S. S; Smetena, I. Determination of Tobacco Alkaloids Using Solid Phase Microextraction and GC-NPD. Chromatographia 1998, 47, 443-448.

11. Cai, J. B.; Liu, B. Z.; Lin, P.; Su, Q. D. Fast Analysis of Nicotine Related Alkaloids in Tobacco and Cigarette Smoke by Megabore Capillary Gas Chromatography. J. Chromatogr. A 2003, 1017, 187-193.

12. Sheng, L. Q.; Ding, L.; Tong, H. W.; Yong, G. P.; Zhou, X. Z.; Liu, S. M. Determination of Nicotine-Related Alkaloids in Tobacco and Cigarette Smoke by GC-FID. Chromatographia 2005, 62, 63-68.

13. Yang, S. S.; Smetena, I.; Huang, C. B. Determination of Tobacco Alkaloids by Gas Chromatography with Nitrogen-Phosphorus Detection. Anal. Bioanal. Chem. 2002, 373, 839-843.

14. Severson, R. F.; Mcduffie, K. L.; Arrendale, R. F. Rapid Method for the Analysis of Tobacco Nicotine Alkaloids. J. Chromatogr. A 1981, 211, 111-121.

15. Lourenço, M. D. G.; Matos, A.; Oliveira, M. C. Gas-Liquid Chromatographic Determination of Major Tobacco Alkaloids. J. Chromatogr. A 2000, 898, 235-243.

16. Wu, W. J.; Ashley, D. L.; Watson, C. H. Determination of Nicotine and Other Minor Alkaloids in International Cigarettes by Solid-Phase Microextraction and Gas Chromatography/Mass Spectrometry. Anal. Chem. 2002, 74, 4878-4884.

17. Shen, J. C.; Shao, X. G. Determination of Tobacco Alkaloids by Gas Chromatography-Mass Spectrometry Using Cloud Point Extraction as a Preconcentration Step. Anal. Chim. Acta 2006, 561, 83-87.

18. Huestes, M. A. Simultaneous Quantification of Methamphetamine, Cocaine, Codeine, and Metabolites in Skin by Positive Chemical Ionization Gas Chromatography-Mass Spectrometry. J. Chromatogr. B 2006, $833,210-218$.

19. Carrizo, D.; Grimalt, J. O. Rapid and Simplified Method for the Analysis of Polychloronaphthalene Congener Distributions in Environmental and Human Samples by Gas Chromatography Coupled to Negative Ion Chemical Ionization Mass Spectrometry. J. Chromatogr. A 2006, 1118 , 271-277.

20. Campora, P.; Bermejo, A. M.; Tabernero, M. J.; Fernandez, P. Use of Gas Chromatography/Mass Spectrometry with Positive Chemical Ionization for the Determination of Opiates in Human Oral Fluid. Rapid Commun. Mass Spectrom. 2006, 8, 1288-1292.

21. Yurchenko, S.; Molder, U. The Determination of Polycyclic Aromatic Hydrocarbons in Smoked Fish by Gas Chromatography Mass Spectrometry with Positive-Ion Chemical Ionization. J. Food. Compos. Anal. 2005, 8, 857-869.

22. Charrois, J. W. A.; Arend, M. W.; Froese, K. L.; Hrudey, S. E. Detecting N-Nitrosamines Water at Nanogram Per Liter Levels Using Ammonia Positive Chemical Ionization. Environ. Sci. Technol. 2004, 38, 4835-4841.

23. He, P.; Guo, Y. L.; Zhu, S. Z. Study on the Reactions of Pyridine with Fluorine-Containing Compounds in the Gas-Phase by Electron Impact/ Fourier Transform Ion Cyclotron Resonance Mass Spectrometry. J. Fluorine Chem. 2005, 126, 107-111.

24. McLuckey, S. A.; Glish, G. L.; Asano, K. G.; Van Berkel, G. J. Self Chemical Ionization in an Ion Trap Mass Spectrometer. Anal. Chem. 1988, 60, 2312-2314.

25. Zhang, F.; Guo, Y. L.; Wei, Q.; Ma, S. M. Study of 2-Iodo-3-(Phenylsulfinyl)2-Propen-1-ol and Its Analogs by Self-Chemical Ionization Mass Spectrometry. Chin. J. Chem. 2001, 19, 851-835.

26. Vairamani, M.; Mirza, U. A.; Srinivas, R. Unusual Positive Ion Reagents in Chemical Ionization Mass Spectrometry. Mass Spectrom. Rev. 1990, 9, 235-258.

27. Westmore, J. B.; Alauddin, M. M. Ammonia Chemical Ionization Mass Spectrometry. Mass Spectrom. Rev. 1986, 5, 381-465.

28. Zhang, X.; Wang, H. Y.; Liao, Y. X.; Ji, H. W.; Guo, Y. L. Study of Methylation of Nitrogen-Containing Compounds in the Gas Phase. J. Mass Spectrom. 2007, 42, 218-224.

29. Ghaderi, S.; Kulkarni, P. S.; Ledford, E. B.; Wilkins, C. L.; Gross, M. L. Chemical Ionization in Fourier Transform Mass Spectrometry. Anal. Chem. 1981, 53, 428-437. 